\title{
IP PATTERNS OF MOVEMENTS IN VSO TYPOLOGY: THE CASE OF ARABIC
}

\author{
C. Belkacemi \\ Manchester Metropolitan University
}

\begin{abstract}
The aim of this article is to explore a particular instantiation of the order in Arabic and to demonstrate that VSO and SVO are frequent alternatives for the language. The article will offer a range of views that have been expressed on the subject in order to give a comparative orientation to this study and will include and cover a range of empirical data in an attempt to discover and to understand the interaction of the IP (Inflectional Phrase) components of the languages underlying system. There will be two kinds of arguments advanced in support of the claim we make with regard to such movement: empirical arguments based on the data provided and theoretical or conceptual arguments based on the internal consistency of the system and the literature that deals with similar issues elsewhere.
\end{abstract}

\section{INTRODUCTION}

It has been argued by Ouhalla (1991: 105) that some languages such as Arabic and Gaelic project a similar surface structure in that they exhibit a similar typological order: VSO. It has also been pointed out that any attempt to classify languages along typological lines should take into consideration the properties of functional categories rather than those of substantives. It is the particular operations which are triggered by movements that result in the language structure being what it is on surface structure. This said, it was established through X-bar theory that $\mathrm{V}$ is Head, and an interesting consequence of this theory is that most nodes dominate no more than two branches, a phenomenon known as binary branching.

For VSO languages, this has the consequence of splitting $\mathrm{V}$ from its complement. It is this characteristic that leads us to investigate whether the subject is base generated as VP internal element and that the alternative order is simply a matter of subject raising or V-lowering, or whether Arabic is underlyingly an SVO language with a surface display of VSO order triggered by a movement which plays a crucial role in not preserving head-initiality. Various views have been expressed on the subject, some of which will be discussed below. 
On the generality of typology, Greenberg (1966) has observed that VSO languages, typically display an alternative word order pattern namely SVO. $\mathrm{He}$ formulates this observation in terms of a generalisation known as Universal 6 reproduced here as: all languages with VSO order have SVO as an alternative. (Celtic languages may be an exception).

Ouhalla (1991: 107, 114 and 1999: 140) attributes this particularity to the fact that although considered to be archetypal examples of VSO languages, Celtic languages nevertheless fail to offer the SVO order alternative that languages like Arabic and Berber offer due to the difference in the nature of their Inflectional categories. Celtic languages are predicted to have AGR inside TNS which is specific to VSO order but instead they display an AGR outside TNS which is essentially the order displayed by SVO languages. This particularity of Inflectional Heads explains why Celtic languages do not offer the alternative SVO order offered by Arabic.

\section{SUBJECT GENERATION}

Koopman and Sportiche (1991) proposed the idea that in VSO languages, the subject is not generated in the specifier of TP but instead it is generated in the specifier of VP under what was called the VP-internal subject hypothesis. This can be interpreted as the result of either V-raising or AGR-lowering.

Assuming Koopman's hypothesis of subject generation to be accurate, the pattern then for the derivation of VSO order must involve a straightforward movement of the verb from its position to that of $\mathrm{T}$ in order to check its agreement while the subject remains in Spec-VP (Carnie 2002: 204-205) resulting in the subject being generated inside the VP as illustrated below.

\section{Figure 1}

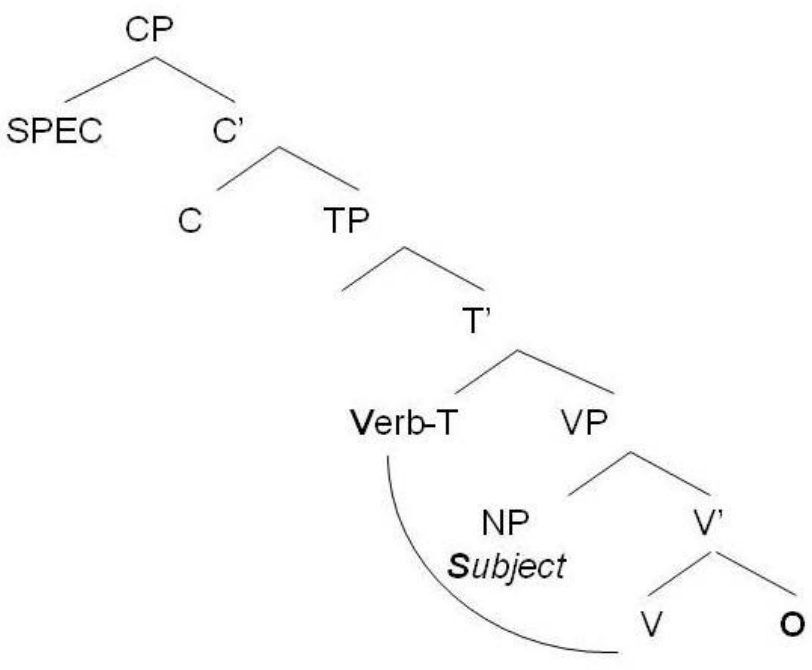


This derives the VSO order as illustrated above and exemplified by the following from Ouhalla:

$1 \quad \rho \alpha ? \alpha \quad \cong \lambda ? \alpha \omega \lambda A: \delta v \zeta \alpha \psi \delta \alpha v$

saw (3 m.s) the- boys (3 m.pl) Nom Zayd

The boys saw Zayd

Ouhalla (1999: 338)

$2 \varphi \alpha ? \alpha \tau \cong \lambda \beta \alpha v A: \tau v$

came ( 3 f.s) the-girls ( 3 f.pl) NOM

The girls came

Ouhalla (1991: 124)

$3 \quad j a ? a \simeq \lambda ? \alpha \omega \lambda A: \delta v$

came (3 m.s) the boys ( 3 m.pl) NOM

The boys came

Ouhalla (1991:125)

Ouhalla (1999: 335), for his part, characterises VSO as the languages where the most natural or unmarked position for the subject is to immediately follow the finite verb. However, there are significant differences in that some VSO languages like Standard Arabic allow SVO alternative order in neutral finite sentences. What is noticeably unusual, compared to standard SVO, is that VP constituents, in particular the verb and its complements, are discontinuous in VSO languages with the subject intervening between them.

Arabic, which is primarily a VSO language, shows that AGR is triggered by movement. This movement can be either of the subject being raised to [spec, AGR] or of the verb being lowered from TP to VP. Following Carnie (2002: 200), most linguists considered VSO languages to simply be exceptions to X-bar theory. They proposed that these languages had a flat structure as illustrated below:

Figure 2

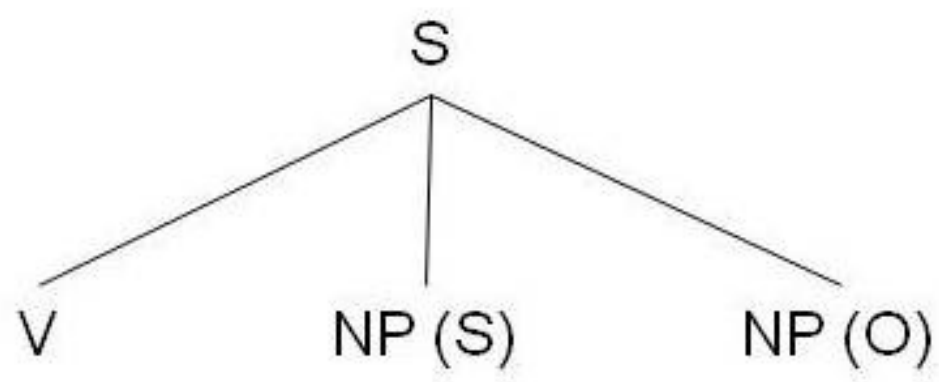


This structure is called flat because there are no hierarchical differences between subjects, objects, and the verb. In other words, there is no structural distinction between complements, adjuncts and specifiers. Unlike SVO where theta theory is fully observed with the single node dominating both the NP which is instead accommodated by the Semantic Selection restrictions.

Since in VSO two nouns (subject and object) are in succession pretty much like the dative movement in English, the hierarchical distinction between them in Arabic is, however, driven by the strong case marking the language exhibits through IP. This results in a much freer order than that of English. Compare 4 to 5 and 6 below:

4 The driver gave the policeman his licence

$$
\begin{aligned}
& 5 \quad \delta \alpha \rho \alpha \beta \alpha \quad \cong \lambda \omega \alpha \lambda \alpha \delta v \cong \lambda ? \alpha \omega \lambda A: \delta \alpha \\
& \eta \iota \tau(3 \mu . \sigma) \tau \eta \varepsilon \beta о \psi(v o \mu) \tau \eta \varepsilon \beta о \psi \sigma \sigma(\alpha \chi \chi)
\end{aligned}
$$

The boy hit the boys

$$
\begin{aligned}
& 6 \quad \delta \alpha \rho \alpha \beta \alpha \cong \lambda \omega \alpha \lambda \alpha \delta \alpha \quad \cong \lambda ? \alpha \omega \lambda A: \delta v \\
& \eta \iota \tau(3 \mu . \sigma) \tau \eta \varepsilon \beta о \psi(\alpha \chi \chi) \tau \eta \varepsilon \beta о \psi \sigma \sigma(v o \mu)
\end{aligned}
$$

The boys hit the boy

It can be noted that two NPs in successive order $(5,6)$ have their cases distinguished regardless of positioning.

\section{MOVEMENT}

Attempts have been made by scholars to explain the difference in typology and the order that results from it. All hypotheses revolved around the question of whether languages are identical at base structure thus making their diversity to be an issue of mere surface structure; or whether there is a deeper gap. Emonds (1980), for instance, proposes that VS (XP) order in VSO languages is derived from raising the verb to $\mathrm{C}$ (via I) from an underlying structure which is identical to that of English in relevant respects. This proposal derives from the universalist idea that all languages have a unique underlying sentence structure and that the difference in order is the result of movements.

Sproat (1985: 336-337), on the other hand, attributes this property of VSO languages to a parameterised restriction on the directionality of nominative case assignment to the subject by finite I. For I to be able to assign case rightward under government, it has to raise to $\mathrm{C}$ located to the left of the subject. Raising of the verb to $\mathrm{C}$ along with finite $\mathrm{I}$ is needed to lexically support I, either for purely morphological reasons or in order to enable finite I 


\section{VOLUME 6}

to assign case: in this instance nominative case to the subject rightward under government.

Mohammad (1989) also posits the argument that the agreement between subjects and AGR in SVO sentences implies that the subject is in a spec-head relation with I and therefore located in Spec-IP as illustrated by the following:

\section{Figure 3}

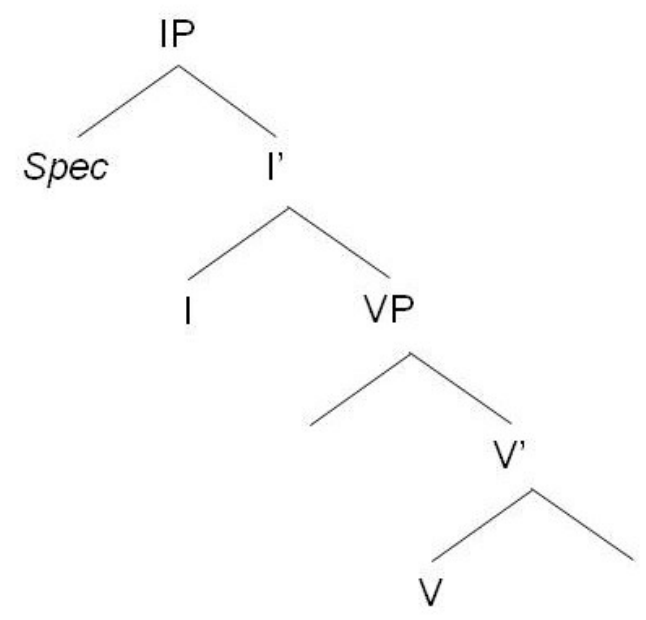

The lack of number agreement between Subject and AGR suggests a VSO order where the subject is not in Spec-head relation with I and therefore not located in Spec-IP. In VSO the subject remains inside VP where it is basegenerated according to the subject inside VP-Hypothesis (Ouhalla 1999: chp.8).

Mohammad's analysis attributes the VSO order to failure on the part of the subject to move to Spec- IP. Apparently such a movement triggers the derivation of the SVO order as indicated by fig. 3 above.

Radford (1997: 320 and 349) also supports the assumption of the VPinternal subject hypothesis in that he posits that subjects originate in spec-VP and raise from Spec VP to spec-TP in order to check their (strong) case feature. Given that a moved constituent leaves behind a trace in any position out of which it moves, it follows that subjects which move from spec VP to spec TP will leave behind a trace in the spec-VP position out of which they move. This, Radford regards as empirical evidence which is essentially similar in character to that used to support the postulation of a PRO subject in control structure. 


\section{Figure.4}

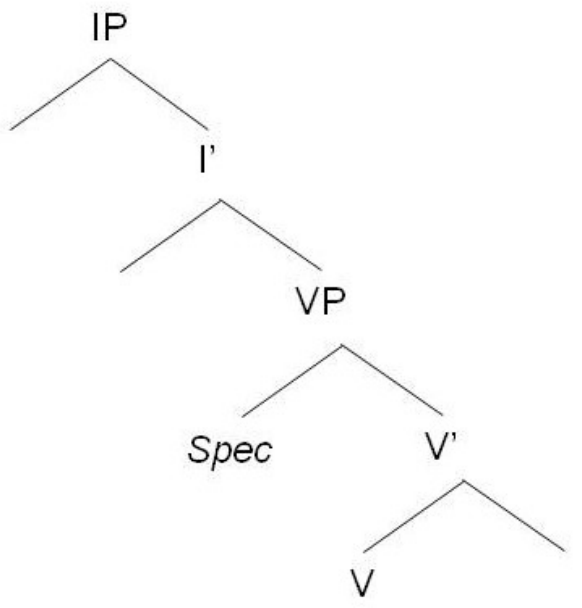

\section{ANALYSIS}

In the light of the various views expressed above, we shall start by exploring the alternative word orders -VSO and SVO- in Arabic and attempt to define the impact AGR and TNS positioning has on the language and its order.

We shall assume a structure where the verb and its complement form a VP and are adjacent at deep-structure (DS). Underlying this attempt is the idea that all languages have a unique underlying sentence structure and that order differences involving specifiers are merely surface phenomena which result from the application of certain movement processes in some languages but not in others (Ouhalla 1999: 336).

For economy and on the grounds that VSO represents only a small percentage in the typology of languages as indicated in Carnie (2002: 202), we shall hypothesise that VSO languages are underlyingly SVO (at DS level) and that the order VSO is derived from SVO through movement.

The question which arises, however, is which movement obtains such order? There are two possible head movements: V- raising or I-lowering. Arabic is a pro-drop language which means that its feature-marking is strong and by necessity the two components of IP (AGR and TNS) have to be overtly expressed. Subsequently it will be a simpler move for $\mathrm{V}$ to raise to IP, than IP (AGR and TNS) to lower to V, and acquire its finite character as illustrated by the following migration from DS to logical form (LF).

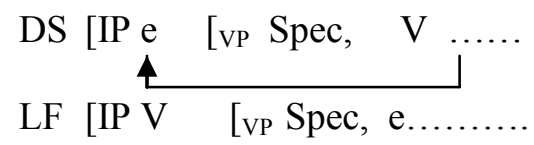




\section{VOLUME 6}

It can be observed that this movement triggers partial agreement involving gender only represented by AGR in the schema below.

$$
\begin{aligned}
& 7 \quad \tau \alpha \delta \rho \imath \beta v \quad \cong \lambda-\beta \imath v \tau v \quad \cong \lambda \omega \alpha \lambda \alpha \delta \alpha \\
& \tau \alpha \delta \rho \imath \beta v(3 \phi \varepsilon \mu . \sigma \imath v \gamma) \quad E \lambda-\beta \imath v \tau v(3 \phi \varepsilon \mu . \sigma \imath v \gamma) \varepsilon \lambda \omega \alpha \lambda \alpha \delta \alpha \\
& \eta \imath \tau(3 \phi \varepsilon \mu . \sigma \imath v \gamma) \tau \eta \varepsilon \gamma \rho \rho \lambda(3 \phi \varepsilon \mu . \sigma \imath v \gamma) \tau \eta \varepsilon \beta o \psi
\end{aligned}
$$

The girl hits the boy

$$
\begin{aligned}
& 8 \quad \tau \alpha \delta \rho \imath \beta v \quad \cong \lambda \beta \alpha v A: \tau v \cong \lambda \omega \alpha \lambda \alpha \delta \alpha \\
& \tau \alpha \delta \rho \imath \beta v(3 \phi \varepsilon \mu . \sigma v \gamma) \quad \varepsilon \lambda \beta \alpha v \alpha: \tau(3 \phi \varepsilon \mu . \pi \lambda v \rho \alpha \lambda) \varepsilon \lambda \omega \alpha \lambda \alpha \delta \alpha \\
& \eta \imath \tau(3 \phi \varepsilon \mu . \sigma \imath v \gamma) \quad \tau \eta \varepsilon \gamma \nu \lambda \sigma(3 \phi \varepsilon \mu . \pi \lambda v \rho \alpha \lambda) \tau \eta \varepsilon \beta o \psi \\
& \text { The girls hit the boy }
\end{aligned}
$$

Although the subjects in 7 and 8 vary in number, the verb remains with a gender exhibit only. Given that no tense agreement is triggered at this stage, we can presume that AGR is lower than TNS in Arabic and split INFL into its 2 components, putting TNS above AGR. The V-features of AGR are invariably strong in standard Arabic with the consequences that the verb raises overtly to AGR. With $\mathrm{V}$ in AGR, failure of the subject to move to [Spec- AGRP] results in the derivation of the VSO order.

\section{Figure.5}

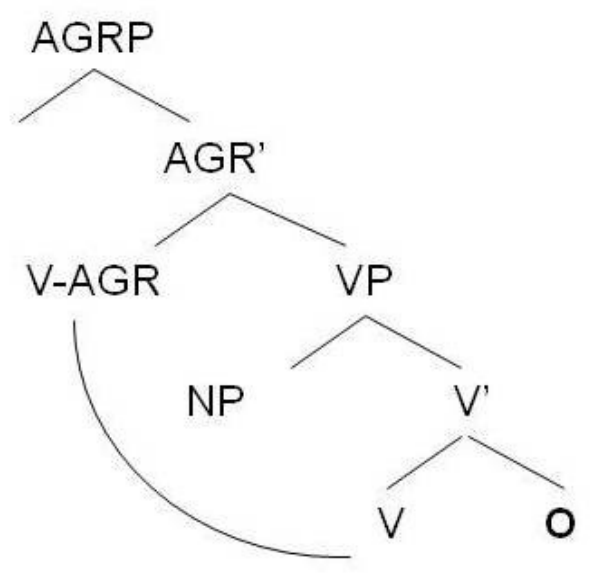

With lexical subjects remaining in post-verbal position in Arabic, this has the consequence of deriving the order VSO at surface structure level. From the INFL perspective, this quite obviously posits the theory that VSO variant in Arabic allows only partial agreement. Until the subject is in a Spec-IP 
relation with I, number agreement with the subject is not expected (Ouhalla 1999: 337-9) as illustrated by the following examples (7 and 8)

$9 \varphi \alpha \delta \rho \imath \beta v \cong \lambda \omega \alpha \lambda \alpha \delta v \cong \lambda \beta \imath v \tau \alpha$

$\varphi \alpha \delta \rho \imath \beta v(3 \mu \alpha \sigma . \sigma l v \gamma) \quad \cong \lambda \omega \alpha \lambda \alpha \delta v(3 \mu \alpha \sigma . \sigma l v \gamma) \cong \lambda \beta \imath v \tau \alpha$

$\eta \imath \tau(3 \mu \alpha \sigma . \sigma \imath v \gamma) \tau \eta \varepsilon \beta o \psi(3 \mu \alpha \sigma . \sigma \imath v \gamma) \tau \eta \varepsilon \gamma l \rho \lambda$

The boy hit the girl

$10 \varphi \alpha \delta \rho \imath \beta v \quad \cong \lambda ? \alpha \omega \lambda \alpha \delta v \cong \lambda \beta \imath v \tau \alpha$

$\varphi \alpha \delta \rho \imath \beta v(3 \mu \alpha \sigma . \sigma v v \gamma) \cong \lambda ? \alpha \omega \lambda \alpha \delta v \quad(3 \mu \alpha \sigma . \pi \lambda v \rho \alpha \lambda) \cong \lambda \beta l v \tau \alpha$ $\eta \imath \tau(3 \mu \alpha \sigma . \sigma \imath v \gamma) \quad \tau \eta \varepsilon \beta o \psi \sigma(3 \mu \alpha \sigma . \pi \lambda v \rho \alpha \lambda) \tau \eta \varepsilon \gamma \iota \rho \lambda$

The boys hit the girl

Now, let us look at SVO order to shed light on other order-related issues.

\section{SVO}

Figure 6. Verb movement

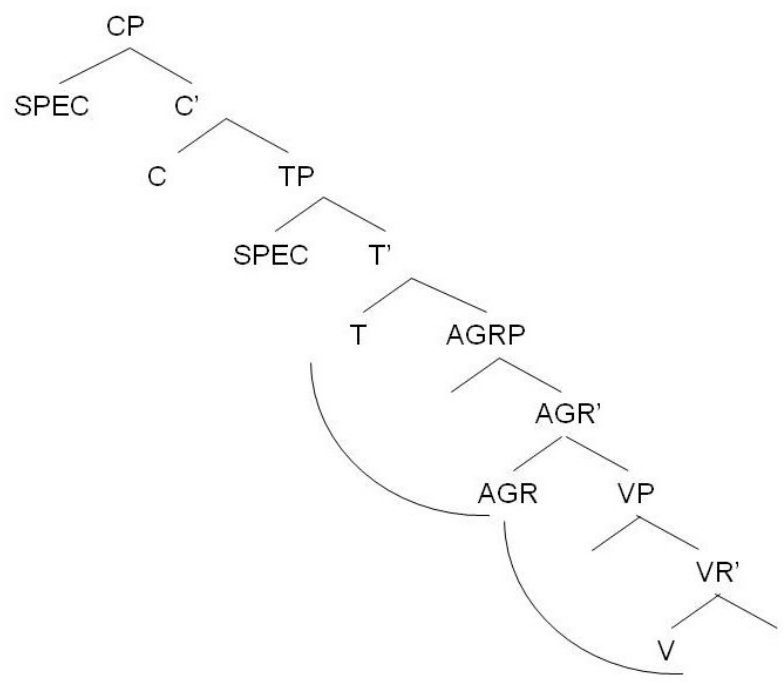

The subject, as indicated earlier, moves to Spec-AGRP resulting in the VSO order. Its present position -between Tense and Agreement- still obstructs the verb from checking its tense inflection which explains the genderagreement only outlined earlier. To enable a complete agreement of the verb with both inflectional heads, another move is required from the subject. This time, it moves from Spec-AGR to Spec-TP, the last of the components of INFL, allowing the verb to move from AGR to T as illustrated above (fig.6). 


\section{VOLUME 6}

There being no obstruction for the verb, the latter moves to TNS to check its inflection and a full AGR and TNS agreement with $\mathrm{V}$ is triggered. The operation is thus considered complete. Verb and Subject moves are illustrated respectively by fig. $6 \mathrm{a}$ above and $6 \mathrm{~b}$ below.

Figure 6 b (spec) Subject movement

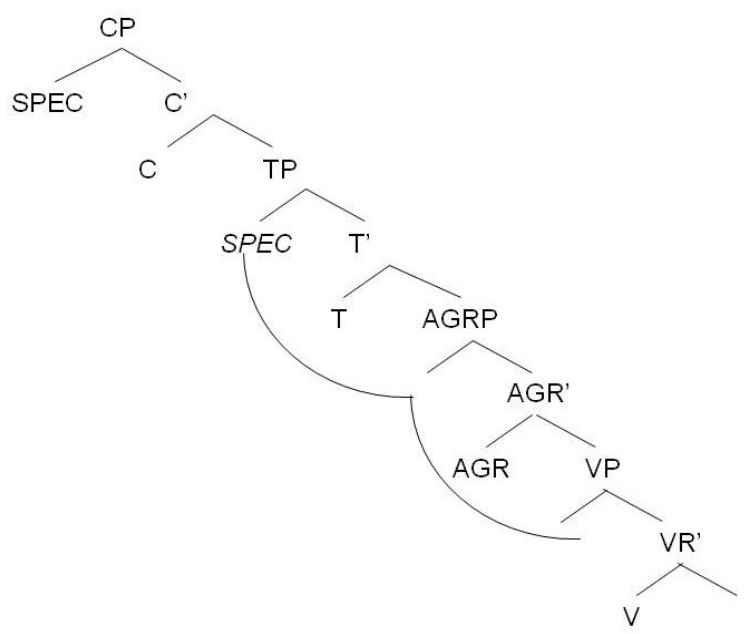

The VSO order can be considered a transitional phase with the verb preceding its subject in movement. When $\mathrm{V}$ is at TP-level, the subject, lower in position, can be assumed in [Spec, AGRP] at LF, thus resulting in the verb showing default agreement features only irrespective of the agreement features of the post-verbal subject as in:

\section{$9 \rho \alpha ? \alpha \alpha \quad \varepsilon \lambda ? \alpha \omega \lambda \alpha \alpha \delta v \quad \zeta \alpha \psi \delta \alpha v$ saw (3m.s) the-boys (3m.pl) Zayd}

The boys saw Zayd

This shows a correlation between order and subject agreement in Standard Arabic while in the VSO order.

The movement of the subject to [Spec- TP] permits the INFL full agreement with the verb, resulting in SVO order as illustrated by the representation below:

$\begin{array}{lrr}10 \quad \varepsilon \lambda ? \alpha \omega \lambda A: \delta v & \rho \alpha ? \alpha v & \zeta \alpha \psi \delta \alpha v \\ \text { the boys (3m.pl) } & \text { saw (3m.pl) } & \text { Zayd } \\ \text { The boys saw Zayd } & \end{array}$


On the evidence of the difference in agreement shown by the lack of agreement in (9) and the full agreement in (10) above, we are compelled to conclude that in Arabic, preverbal subjects do occupy the Spec position of TP and show its marking (10), but in post-verbal instances, it can be implied that the subject is in a rather different position and is not governed by TP but AGRP, hence the lack of marking (10).

\section{CONCLUSION}

Arabic, like all languages with strong INFL marking has a flexible order. It exhibits VSO mainly in Standard forms and SVO in Modern spoken forms. There seems to be a correlation between the order of AGR/ TNS and the surface position of the subject. When the subject is in [Spec-VP] for the verb to check its INFL, it has to move to the lowest part of INFL which is AGR and collects its inflection. However, the resulting subject-verb agreement is weak and remains confined to gender only. The order is VSO.

Tense, higher than AGR, remains still missing from the verb inflection. This triggers another subject movement in order to allow $\mathrm{V}$ to reach it. The subject in accordance with the principle of locality moves up to the nearest location TP and gets positioned in [Spec-TP]. There being no obstruction, the verb moves after it and checks the remaining part of its inflection. Now that the subject is in [Spec-TP] there is full agreement with the verb bearing both TNS and AGR marking and the order is SVO.

\section{REFERENCES}

Carnie, A. (2002) Syntax. Blackwell Publishing, Oxford.

Emonds, J.E (1980) Word Order in generative grammar. Journal of Linguistic research 1, pp.33-54.

Greenberg, Joseph H. 1966. "Some Universals of Grammar with Particular Reference to the Order of Meaningful Elements. Universals of Language, ed. by J.H. Greenberg. Cambridge, Massachussets, pp.73-113

Koopman, H. and Sportiche, D. (1991) The position of subjects. Lingua 85, Special issue on VSO Languages.

Mohammad, M.A (1989) The Sentential Structure of Arabic. Doctoral dissertation. University of Southern California, Los Angeles.

Ouhalla, J. (1991) Functional Categories and Parametric Variation. Routledge, London.

Ouhalla, J. (1999) Introducing Transformational Grammar, Arnold, London.

Radford, A. (1997) Syntactic Theory and the Structure of English, a Minimalist Approach, Cambridge University Press.

Schlonsky, U (1997) Clause structure and Word Order in Hebrew and Arabic, An Essay in Comparative Syntax, Oxford Studies in Comparative Syntax, Oxford University Press. 
VOLUME 6

Sproat, R. (1985) Welsh Syntax and VSO structure. Natural Language and Linguistic Theory 3, pp.173-216.

Vikner, S (1997) V' to I' movement and Inflection for person in all tenses in

Liliane Haegeman (ed). The New comparative Syntax Longman, Harlow, Essex, pp.189-213.

Webelhuth, G (1995) Government and Binding Theory and the Minimalist Program. Blackwell, Cambridge, Massachussetts. 